\title{
Construction of a novel selection system for endoglucanases exhibiting carbohydrate-binding modules optimized for biomass using yeast cell-surface engineering
}

\author{
Akihito Nakanishi, Jungu Bae, Kouichi Kuroda and Mitsuyoshi Ueda*
}

\begin{abstract}
To permit direct cellulose degradation and ethanol fermentation, Saccharomyces cerevisiae BY4741 ( $\Delta$ sed1) codisplaying 3 cellulases (Trichoderma reesei endoglucanase II [EG], T. reesei cellobiohydrolase II [CBH], and Aspergillus aculeatus $\beta$-glucosidase I [BG]) was constructed by yeast cell-surface engineering. The EG used in this study consists of a family 1 carbohydrate-binding module (CBM) and a catalytic module. A comparison with family 1 CBMs revealed conserved amino acid residues and flexible amino acid residues. The flexible amino acid residues were at positions $18,23,26$, and 27 , through which the degrading activity for various cellulose structures in each biomass may have been optimized. To select the optimal combination of CBMs of EGs, a yeast mixture with comprehensively mutated CBM was constructed. The mixture consisted of yeasts codisplaying EG with mutated $\mathrm{CBMs}$, in which 4 flexible residues were comprehensively mutated, $\mathrm{CBH}$, and $\mathrm{BG}$. The yeast mixture was inoculated in selection medium with newspaper as the sole carbon source. The surviving yeast consisted of RTSH yeast (the mutant sequence of CBM: N18R, S23T, S26S, and T27H) and wild-type yeast (CBM was the original) in a ratio of 1:46. The mixture (1 RTSH yeast and 46 wild-type yeasts) had a fermentation activity that was 1.5 -fold higher than that of wild-type yeast alone in the early phase of saccharification and fermentation, which indicates that the yeast mixture with comprehensively mutated CBM could be used to select the optimal combination of CBMs suitable for the cellulose of each biomass.
\end{abstract}

Keywords: Biorefinery, Carbohydrate-binding module (CBM), Cellulase, Yeast cell-surface engineering

\section{Introduction}

Recently, the production of energy without fossil resources has become necessary to establish a sustainable society because of expanded energy demand, limitations of oil drilling, and environmental pollution (Ge et al. 2011; Gerngross and Slater 2000; Stöcker 2008). Biorefinery is an important concept because it uses the biomass in a natural cycle (Bouaid et al. 2010; FitzPatrick et al. 2010; López et al. 2010). Bioethanol is a popular energy resource in biorefinery; however, current biorefineries mainly use grain biomass to produce biofuels, which competes with food supply (Ferreira et al. 2010). Thus, use of biomass garbage has attracted attention for biorefineries. Biomass garbage is

\footnotetext{
* Correspondence: miueda@kais.kyoto-u.ac.jp

Division of Applied Life Sciences, Graduate School of Agriculture, Kyoto University, Sakyo-ku, Kyoto 606-8502, Japan
}

an important resource to produce bioethanol because it is mainly composed of cellulose as a carbon source (Chandel and Singh 2011; Wu et al. 2011). However, most cellulosedegrading techniques require expensive infrastructure, intensive energy, hard chemicals, and the separation of saccharification and fermentation processes. Thus, innovation is necessary to solve these problems.

Yeast cell-surface engineering enables $10^{4}-10^{5}$ enzymes to be displayed on the surface of a yeast cell (Kuroda et al. 2001, 2011; Lin et al. 2003; Nishitani et al. 2010; Shibasaki et al. 2001; Washida et al. 2001; Ye et al. 2000). The displaying yeast can be used as a whole-cell biocatalyst without requiring enzyme-separation and -purification processes, and it can immediately take in glucose degraded from cellulose; thus, it is hardly contaminated by other organisms. Furthermore, direct ethanol fermentation from cellulose 
could be conducted by using cellulase-displaying yeast (Fujita et al. 2002, 2004; Murai et al. 1998).

Cellulases mainly consist of endoglucanase (EG), cellobiohydrolase (CBH), and $\beta$-glucosidase (BG) (Hess et al. 2011; Todaka et al. 2010). EG degrades cellulose at endo points, and degradation by EG is important in the early phase of cellulose degradation. The degrading activity of EG is largely supported by the carbohydrate-binding module (CBM), which is matched to the cellulose in each biomass (Ståhlberg et al. 1991). Furthermore, cellulose degradation is efficiently supported by CBMs because EGs with different CBMs have different binding specificities; CBM is probably required for each biomass in the degrading phase. Thus, it should be possible to improve the cellulase activity when a suitable combination of CBMs is optimized for each cellulose.

In many CBM families, the family $1 \mathrm{CBM}$ is important because it can bind to crystalline cellulose in the early degrading phase, which is important to promote cellulose degradation (Fujita et al. 2004). Thus far, most studies have focused on 3 conserved amino acid residues to improve the binding ability of the CBM (Fukuda et al. 2006). In contrast, flexible amino acid residues have rarely been investigated, even though these residues may affect the binding specificity for the cellulose of each biomass.

In this study, the amino acid sequences of family 1 CBMs were compared, and the flexible sequences were determined. To select the optimal combination of CBMs of Trichoderma reesei EG IIs for cellulose degradation, a yeast mixture with comprehensively mutated CBM was constructed. The mixture consisted of yeasts codisplaying EG with mutated CBM, in which 4 flexible residues were comprehensively mutated, $T$. reesei CBH II, and Aspergillus aculeatus BG I. The yeast mixture was first inoculated into the selection medium with newspaper as a sole carbon source. After selection, the combination of yeasts displaying EG with mutated CBM, $\mathrm{CBH}$, and $\mathrm{BG}$ was applied to direct fermentation of newspaper.

\section{Materials and methods}

\section{Strains and media}

Escherichia coli strain DH5 $\alpha\left(\mathrm{F}^{-}, \varphi 80 \mathrm{~d} l a c Z \Delta \mathrm{M} 15, \Delta[\right.$ lacZYA-argF]U169, endA1, hsdR17[ $\left.r_{k}^{-}, m_{k}^{+}\right]$, supE44, thi-1, $\lambda-$, recA1, gyrA96, relA1, deoR) was used as a host for recombinant DNA manipulation and grown in Luria-Bertani medium $(1 \%[w / v]$ tryptone, $0.5 \%[w / v]$ yeast extract, and $1 \%[w / v]$ sodium chloride) containing $100 \mu \mathrm{g} / \mathrm{mL}$ ampicillin.

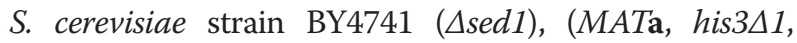
leu2 $\Delta 0$, met15 $\Delta 0$, ura3 $\Delta 0$, YDR077w::KanMX4), which was obtained from EUROSCARF (Frankfurt, Germany), was used for the cell surface display of cellulase. Yeast transformants were aerobically cultured in synthetic- dextrose (SD) medium $(0.67 \%[w / v]$ yeast nitrogen base without amino acids, $2 \%[w / v]$ glucose, and $0.003 \%[w / v]$ L-methionine) as pre-incubation medium and in SD with casamino acids $(\mathrm{SDC})$ medium $(0.67 \%[w / v]$ yeast nitrogen base without amino acids, $2 \%[w / v]$ glucose, $0.5 \%[w / v]$ casamino acids, $0.003 \%[w / v]$ L-methionine) as main incubation medium for saccharification and fermentation.

Optimal combination of yeasts displaying EG with mutated CBM was selected from the yeast mixture with comprehensively mutated CBM in a yeast nitrogen base $(0.67 \%[w / v]$ yeast nitrogen base without amino acids and $0.003 \%[w / v]$ L-methionine) with $1 \%$ newspaper (YNB-newspaper medium).

Yeast fermentation was conducted in $50 \mathrm{mM}$ citric acid buffer ( $\mathrm{pH}$ 5.0) containing a yeast nitrogen base with casamino acids $(0.67 \%[w / v]$ yeast nitrogen base without amino acids, $0.5 \%[\mathrm{w} / \mathrm{v}]$ casamino acids and $0.003 \%[w / v]$ L-methionine) with $0.4 \%[w / v]$ laccasetreated newspaper. The medium was termed "yeast nitrogen base with casamino acid (YNBC) medium" .

\section{Preparation of laccase-treated newspaper}

Newspaper was sterilized with $99.5 \%$ ethanol (Nakanishi et al. 2012b), and the volume of the ethanol was 20-fold of the newspaper mass. After sterilization, the newspaper was dried and treated with laccase DAIWA Y120 (4.3 $\mathrm{POU} / \mathrm{mL}$ ) in $50 \mathrm{mM}$ sodium acetate buffer ( $\mathrm{pH}$ 5.0) for $24 \mathrm{~h}$ as pretreatment. The newspaper was then washed with sterilized water and dried for fermentation.

\section{Plasmid construction}

Three plasmids to display EG (pEG), CBH (pCBH), and $\mathrm{BG}$ (pBG) were constructed in the previous study (Nakanishi et al. 2012a,b).

\section{Determination of the flexible amino-acid residues in family 1 CBMs}

The amino acid sequences of 92 family 1 CBMs were compared. All sequence data of family $1 \mathrm{CBMs}$ were obtained from BLAST (http://blast.ncbi.nlm.nih.gov/Blast.cgi). After comparison, the amino acid residues for which the most frequent amino acid appeared with a frequency of less than $40 \%$ were selected as the flexible amino-acid residues (Table 1).

\section{Construction of a yeast mixture codisplaying EG with comprehensively mutated $\mathrm{CBM}, \mathrm{CBH}$, and BG}

All primers used for plasmid construction are listed in Table 2.

To construct the yeast mixture with comprehensively mutated CBM, the flexible amino acid residues (i.e., those at positions 18 of N, 23 of S, 26 of S, and 27 of T) were comprehensively mutated by yeast homologous recombination. First, the 2 double-stranded DNA fragments, i.e., 
Table 1 Comparison of CBM amino-acid sequences of EG in family 1 CBMs (92 samples)

\begin{tabular}{|c|c|c|}
\hline No. & A.A. & remarks column \\
\hline 5 & W & $W(61 \%), Y(39 \%)$ \\
\hline 6 & G & $\mathrm{G}(91 \%), \mathrm{QA}(3 \%), \mathrm{Y}(2 \%)$ \\
\hline 7 & Q & Unchanging \\
\hline 8 & $\mathrm{C}$ & Unchanging \\
\hline 9 & G & Unchanging \\
\hline 10 & G & Unchanging \\
\hline 11 & । & I(52\%), Q(20\%), S(14\%), N(7\%), T(4\%), ARL(1\%) \\
\hline 12 & G & $\mathrm{G}(92 \%), \mathrm{N}(9 \%), \mathrm{S}(1 \%)$ \\
\hline 13 & W & $W(74 \%), Y(23 \%), F(3 \%)$ \\
\hline 14 & $\mathrm{~S}$ & $\mathrm{~T}(52 \%), \mathrm{S}(42 \%), \mathrm{N}(3 \%), \mathrm{IK}(1 \%)$ \\
\hline 15 & G & Unchanging \\
\hline 16 & $\mathrm{P}$ & $P(74 \%), A(17 \%), S(4 \%), L(3 \%), Q(2 \%)$ \\
\hline 17 & $\mathrm{~T}$ & Unchanging \\
\hline$\underline{18}$ & $\mathrm{~N}$ & $\mathrm{~T}(28 \%), \mathrm{N}(13 \%), \mathrm{S}(12 \%), \mathrm{AV}(11 \%), \mathrm{QA}(5 \%), \operatorname{RECT}(3 \%), \mathrm{DKI}(1 \%)$ \\
\hline 19 & C & Unchanging \\
\hline 20 & A & $A(49 \%), V(36 \%), Q(5 \%), E T(4 \%), D(2 \%)$ \\
\hline 21 & $\mathrm{P}$ & $S(66 \%), A(21 \%), P(8 \%), T(4 \%), G(1 \%)$ \\
\hline 22 & G & $G(86 \%), P(13 \%), S(1 \%)$ \\
\hline$\underline{23}$ & $\mathrm{~S}$ & $S(35 \%), A(25 \%), T(17 \%), Y(12 \%), F N L(3 \%), W G(1 \%)$ \\
\hline 24 & A & $T(67 \%), A(14 \%), V(9 \%), C(5 \%), S(3 \%), K(2 \%)$ \\
\hline 25 & C & Uchanging \\
\hline$\underline{26}$ & $S$ & $\mathrm{~T}(27 \%), \mathrm{Q}(24 \%), \mathrm{S}(22 \%), \mathrm{V}(10 \%), \mathrm{H}(8 \%), \mathrm{K}(4 \%), \mathrm{AE}(2 \%), \mathrm{M}(1 \%)$ \\
\hline$\underline{27}$ & $\mathrm{~T}$ & $V(38 \%), T(20 \%), K(17 \%), S(8 \%), Y(5 \%), A E(4 \%), I R Q H(1 \%)$ \\
\hline 28 & $\mathrm{~L}$ & L(53\%), Q(13\%), YV(8\%), I(6\%), S(5\%), T(3\%), GAWM(1\%) \\
\hline 29 & $\mathrm{~N}$ & $N(97 \%), S(2 \%), G(1 \%)$ \\
\hline 30 & $P$ & $P(56 \%), D(25 \%), A S(5 \%), Q E(4 \%)$ \\
\hline 31 & Y & $Y(75 \%), W(22 \%), F(2 \%), A(1 \%)$ \\
\hline 32 & Y & $Y(99 \%), H(1 \%)$ \\
\hline 33 & A & $S(71 \%), A(20 \%), Y(4 \%), H(3 \%), F(2 \%)$ \\
\hline 34 & Q & Uchanging \\
\hline 35 & C & Uchanging \\
\hline
\end{tabular}

Amino-acid numbers (No.) and sequences (A.A.; 1-letter-notation) of the CBM of EG; remarks column, the results of amino-acid comparisons in family $1 \mathrm{CBM}$; underline: the flexible residue (the conservation $<40 \%$ ).

the insert and the vector, were amplified by polymerase chain reaction (PCR). A single-stranded DNA fragments that included NNK sequences $(\mathrm{N}=\mathrm{A}$ or $\mathrm{C}$ or $\mathrm{G}$ or $\mathrm{T} ; \mathrm{K}=$ $\mathrm{G}$ or $\mathrm{T}$ ) at the flexible amino acid residues of the CBM of EG were synthesized and amplified as a double-stranded DNA fragment using the NNKrev primer via the Klenow fragment (Toyobo, Osaka, Japan). The DNA fragments were extended with the EGct F1 primer and EGct R1 primer, and further extended with the EGct F2 primer and EGct R2 primer; the product was then used as the insert fragment. The linearized pEG without the flexible amino
Table 2 Primers to construct the comprehensively mutated CBM and to confirm the mutated CBM-sequences

\begin{tabular}{ll}
\hline Primer & Sequence \\
\hline pEGout F & 5'-CGTAGGTCCGCTCCAACCAATACC-3' \\
pEGout R & 5'-CTCAATCCTATTATGCGCAATGTATTCCG-3' \\
NNK primer & 5'-TTGGAGCGGACCTACGNNKTGTGCTCCTGGCNNKGCTT \\
& CTNNKNNKCTCAATCCTTATTATGCGCAATGT-3' \\
NNK rev & 5'-ACATTGCGCATAATAAGGATTGAG-3' \\
EGct F1 & 5'-CCAGTGTGGAGGTATTGGTTGGAGCGGACCTACG-3' \\
EGct R1 & 5'-GTCGAAGTGGTGATAGTAGTGGCTCCCGGAATACATTG \\
& CGCATAATAAGGATGAG-3' \\
EGct F2 & 5'-ATAGATCTCAGCAGACTGTCTGGGGCCAGTGTGGA \\
& GGTATTGG-3' \\
EGct R2 & 5'-GCACGCGTGGTGGTGGTTGGACCGGATGGTGGCCGGG \\
TCGAAGTGGTGATAGTAGTGGC-3' & 5'-CATGCAACTGTTCAATTGGCATTG-3' \\
EGII360bp & 5'-AGGATAAACCTTCGAGGTAACGCAAGTGCC-3' \\
rev & \\
\hline
\end{tabular}

acid-encoding region in the CBM of EG was amplified with the pEGout $\mathrm{F}$ primer and pEGout R primer, and the product was used as the vector fragment. The insert fragments and the vector fragment were simultaneously introduced into yeast codisplaying $\mathrm{CBH}$ and $\mathrm{BG}$ for homologous recombination. The transformants were named as the yeast mixture with comprehensively mutated CBM.

\section{Yeast transformation}

Plasmid introduction into BY4741 ( $\Delta$ sed1) cells was performed using the lithium acetate method (Ito et al. 1983) with a YEASTMAKER yeast transformation system (Clontech Laboratories, CA, USA). The transformants were isolated on a selective $\mathrm{SD}$ medium plate at $30^{\circ} \mathrm{C}$ for $2-3$ days.

\section{Selection of optimal combination of CBMs}

Optimal combination of yeasts displaying EG with mutated CBMs was selected from the yeast mixture with comprehensively mutated CBMs in a medium that contained biomass cellulose (newspaper etc.) as a sole carbon source (Figure 1). In this study, the yeast mixture with comprehensively mutated CBM was incubated in YNB-newspaper medium for 1 week twice in a row. The yeast and the newspaper were then transferred to SD+M agarose plates to isolate the surviving yeasts (Figure 2). The CBM-encoding DNAs of the surviving yeasts were amplified by colony-direct PCR using the CHK primer and EGII360bp rev primer (Table 2) and sequenced after purification by using the ethanol-precipitation method.

\section{Immunofluorescence labeling of yeast cells and observation}

Immunofluorescence labeling of cells was performed according to the previously described method (Kobori et al. 


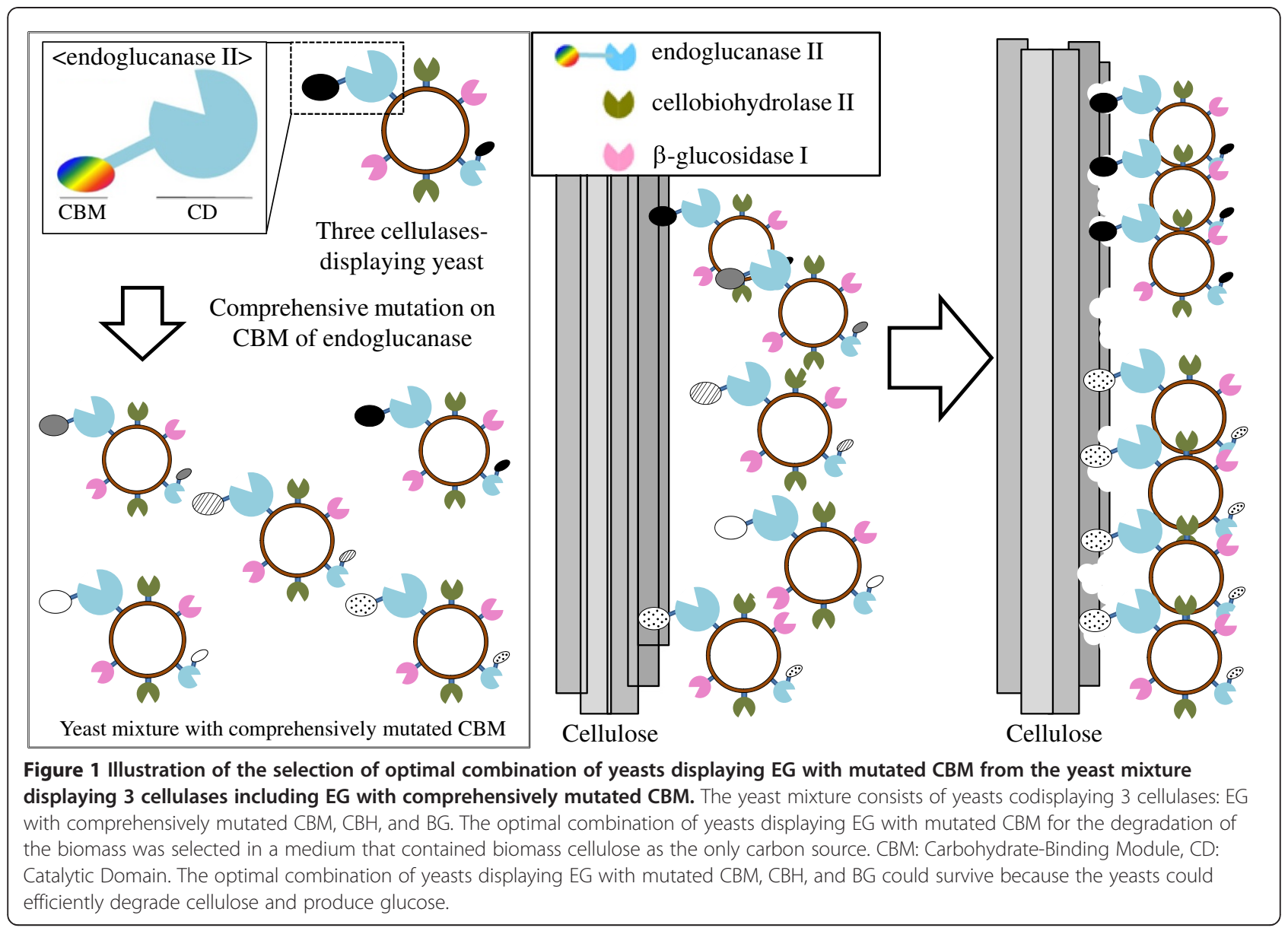

1992). Three monoclonal antibodies were used as the primary antibodies at a dilution of 1:300: mouse monoclonal anti-FLAG M2 antibody (Sigma-Aldrich, MO, USA) for EG, mouse monoclonal StrepMAB-Classic antibody (IBA, MO, USA) for $\mathrm{CBH}$, and mouse monoclonal anti-RGS-His antibody (QIAGEN, Hilden, Germany) for BG. The mixtures of cells and the respective antibodies were incubated at room temperature with gentle shaking for $1.5 \mathrm{~h}$, and the cells were washed with phosphate-buffered saline (PBS; pH 7.4). As the secondary antibody, Alexa Fluor 488 anti- mouse IgG (Invitrogen, CA, USA) was used at a dilution of 1:300 at room temperature with gentle shaking for $1.5 \mathrm{~h}$. The cells were washed with PBS ( $\mathrm{pH} 7.4)$ and observed with an inverted microscope IX71 (Olympus, Tokyo, Japan) through a U-MNIBA2 mirror unit with a BP470-490 excitation filter, a DM505 dichroic mirror, and a BA510-550 emission filter (Olympus). Live images were obtained using Aqua Cosmos 2.0 software (Hamamatsu Photonics, Shizuoka, Japan) to control a digital changecoupled device camera (Hamamatsu Photonics).

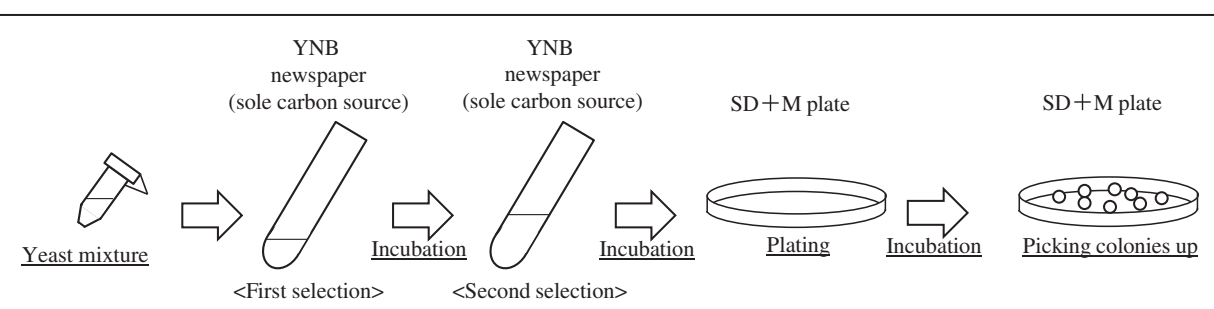

Figure 2 Scheme to select optimal combination of yeasts displaying EG with mutated CBM. The selection was conducted twice using the yeast mixture displaying 3 cellulases including EG with comprehensively mutated CBM in a medium containing laccase-treated newspaper as the sole carbon source. After selection, the medium containing the yeast and newspaper was directly transferred to SD+M medium plate. The surviving yeasts were obtained for sequencing. 


\section{Measurement of yeast fermentation activity}

After pre-cultivation in SD medium for $48 \mathrm{~h}$ at $30^{\circ} \mathrm{C}$, each yeast cell was aerobically cultivated for $60 \mathrm{~h}$ at $30^{\circ} \mathrm{C}$ in SDC medium. The cells were collected by centrifugation for $5 \mathrm{~min}$ at $5000 \times g$ and $4^{\circ} \mathrm{C}$, and washed with PBS $(\mathrm{pH}$ 7.4). To confirm the result of the selection for the newspaper from the yeast mixture with comprehensively mutated CBM, RTSH yeast (4 amino acid residues of the CBM were mutated: N18R, S23T, S26S, and T27H) and wild-type yeast (the CBM was not mutated) were mixed in a ratio of 1:46 and a total $\mathrm{OD}_{600}$ of 10 in YNBC medium. The yeasts were termed "blended yeasts". $\mathrm{CO}_{2}$ gas was injected into the reaction vessel for $2 \mathrm{~min}$ to displace $\mathrm{O}_{2}$. For fermentation, the blended yeasts were semi-aerobically cultured in YNBC medium stirred by a magnetic bar rotating at $130 \mathrm{rpm}$ at $30^{\circ} \mathrm{C}$. Five hundred microliters of reacting medium was collected and filtered using Ultrafree-MC Centrifugal Filter Units (Millipore, MA, USA) for ethanol quantification. The produced ethanol was quantified by using a high-performance liquid chromatography (HPLC) system that consisted of a LC-20 AD pump (Shimadzu, Kyoto, Japan), a CTO-20A column oven (Shimadzu), a RID-10A detector (Shimadzu), a YMC-Pack Polyamine II column $(4.6 \times 250 \mathrm{~mm})$ (YMC Co. Ltd., Kyoto, Japan), and a 7725 injector (Rheodyne, CA, USA). The concentration of produced ethanol was determined from the chromatographic data monitored by the RID$10 \mathrm{~A}$, and the results were processed using LC Solution software (Shimadzu). The mobile phase was water and acetonitrile mixed in a ratio of 5:95 as isocratic, and the temperature of the column oven was set at $30^{\circ} \mathrm{C}$. The flow rate was $1.0 \mathrm{~mL} / \mathrm{min}$.

\section{Results}

\section{Comparison of the sequences of family 1 CBMs}

Conserved and flexible amino acid residues were determined by comparing the amino acid sequences of 92 types of family 1 CBMs (Table 1). More than $99 \%$ of the 5th, 31st, and 32nd amino acids of the CBMs were aromatic amino acids that bind to the flat surface of crystalline cellulose; the 7th, 8th, 9th, 10th, 15th, 17th, 19th, 25th, 34th, and 35th amino acids were conserved as skeletal elements, where the 19th and 35th amino acids as well as the 8th and 25th amino acids formed disulfide bonds with each other without any exception. The frequency of appearance of the major amino acids at the 18th, 23rd, 26th, and 27th positions was lower than $40 \%$, and we decided that they were flexible amino acid residues.

\section{Construction of a yeast mixture with comprehensively mutated CBM}

To construct the yeast mixture with comprehensively mutated CBM, the flexible amino acid residues were comprehensively mutated. The DNA sequences focused on the 18th, 23rd, 26th, and 27th amino acids were evaluated to confirm the construction (Table 3). We concluded that the sequence of the CBM region in EG was comprehensively mutated because the null hypothesis for the appearance of each amino acid was rejected (appearance value $<0.05$ ).

\section{Screening of optimal combination of CBMs}

We performed screening to select the optimal combination of yeasts displaying EG with mutated CBM from the yeast mixture with comprehensively mutated CBM using newspaper as the sole carbon source. After the incubation of the yeast mixture in the medium containing newspaper, yeasts were spread on a $\mathrm{SD}+\mathrm{M}$ plate. Colonies were formed by the surviving yeast after 2-3 days, and the CBM-encoding DNAs in the surviving yeasts on the plate were sequenced. The surviving yeasts consisted of RTSH yeast (the sequence of the CBM of EG among displayed 3 cellulases was mutated: N18R, S23T, S26S, and $\mathrm{T} 27 \mathrm{H}$ ) and wild-type yeasts (the sequence of the CBM of EG was not changed) in a ratio of 1:46. These results suggest that the combination of RTSH yeast and wild-type yeast could be optimal for the degradation of cellulose in newspaper.

\section{Confirmation of the display of EG with mutated CBM, $\mathrm{CBH}$, and $\mathrm{BG}$}

Immunofluorescence labeling was performed with the 3 different antibodies to confirm the display of cellulases including EG with mutated CBM on the yeast cell surface. The green fluorescence of Alexa Fluor 488 for each tag was observed on the cell surface of each transformant (Figure 3), and each cellulase was co-displayed on the yeast cell surface (data not shown). This result indicated that the 3 cellulases (EG with mutated CBM, $\mathrm{CBH}$, and $\mathrm{BG}$ ) were successfully displayed on the surface of RTSH yeast.

\section{Fermentation activity of the mixture of RTSH yeast and wild-type yeast}

To confirm the result of screening that the combination of RTSH and wild-type yeast in a ratio of 1:46 is optimal for degradation of newspaper, we performed direct fermentation of newspaper. The fermentation activities of the blended yeast (the combination of RTSH yeast and wild-type yeast), RTSH yeast alone, and wildtype yeast alone were compared (Figure 4). The activity of the blended yeast was 1.48-fold higher than that of wild-type yeast at $6 \mathrm{~h}$. The activity of RTSH yeast was almost equal to that of wild-type yeast at $6 \mathrm{~h}$; however, the activity of RTSH yeast was 1.21-fold higher than that of wild-type yeast at $24 \mathrm{~h}$. These results indicate that the saccharification activity of blended yeast which 
Table 3 Confirmation of appearance of amino acids in comprehensively mutated CBM in 88 samples

\begin{tabular}{|c|c|c|c|c|c|c|c|}
\hline \multicolumn{2}{|c|}{ A.A. number; 18} & \multicolumn{2}{|c|}{ A.A. number; 23} & \multicolumn{2}{|c|}{ A.A. number; 26} & \multicolumn{2}{|c|}{ A.A. number; 27} \\
\hline A.A. & Rate & A.A. & Rate & A.A. & Rate & A.A. & Rate \\
\hline $\mathrm{T}$ & $10(11.4 \%)$ & S & $12(13.6 \%)$ & L & 10 (11.4\%) & $\mathrm{L}$ & 11 (12.5\%) \\
\hline S & 9 (10.2\%) & G & $9(10.2 \%)$ & V & $8(9.1 \%)$ & $\mathrm{R}$ & 9 (10.2\%) \\
\hline A & 9 & L & 7 (8.0\%) & $\mathrm{S}$ & 8 & S & $8(9.1 \%)$ \\
\hline L & $7(8.0 \%)$ & $\mathrm{T}$ & 7 & $\mathrm{R}$ & $7(8.0 \%)$ & $\mathrm{T}$ & 7 (8.0\%) \\
\hline$P$ & 7 & $\mathrm{R}$ & 7 & W & $6(6.8 \%)$ & G & $6(6.8 \%)$ \\
\hline F & $6(6.8 \%)$ & A & $6(6.8 \%)$ & I & $5(5.7 \%)$ & C & $5(5.7 \%)$ \\
\hline R & 6 & M & 6 & Stop & 5 & $P$ & 5 \\
\hline E & $5(5.7 \%)$ & W & $5(5.75)$ & G & 5 & W & $4(4.5 \%)$ \\
\hline Y & $4(4.55)$ & $\mathrm{H}$ & $4(4.5 \%)$ & $\mathrm{T}$ & $4(4.5 \%)$ & V & 4 \\
\hline V & $3(3.4)$ & Q & 4 & $P$ & 4 & A & $3(3.4 \%)$ \\
\hline Stop & 3 & $N$ & $3(3.4 \%)$ & Y & 4 & $\mathrm{~F}$ & 3 \\
\hline $\mathrm{N}$ & 3 & D & 3 & A & $3(3.4 \%)$ & $\mathrm{D}$ & 3 \\
\hline K & 3 & I & 3 & M & 3 & M & 3 \\
\hline$C$ & $2(2.3 \%)$ & $\mathrm{F}$ & $2(2.3 \%)$ & $\mathrm{F}$ & 3 & $\mathrm{H}$ & 3 \\
\hline W & 2 & K & 2 & C & 3 & K & 3 \\
\hline G & 2 & V & 2 & $N$ & $2(23 \%)$ & Stop & 3 \\
\hline Q & 2 & P & 2 & $E$ & 2 & I & $2(2.3 \%)$ \\
\hline I & 2 & Y & 2 & Q & 2 & Q & 2 \\
\hline D & 2 & $E$ & 1 (1.1\%) & K & 1 (1.1\%) & Y & 2 \\
\hline \multirow[t]{3}{*}{ M } & 1 (1.1\%) & Stop & 1 & $\mathrm{H}$ & 1 & $N$ & $1(1.1 \%)$ \\
\hline & & & & $\mathrm{F}$ & 1 & $E$ & 1 \\
\hline & & & & $\mathrm{D}$ & 1 & & \\
\hline
\end{tabular}

we obtained from screening was higher than that of wild-type yeast alone and the screening system is suitable for selection of optimal combination of CBMs.

\section{Discussion}

In this study, 92 amino acid sequences of family 1 CBMs were compared, and conserved and flexible sequences were determined (Table 1). The conserved sequences contained components of the peptide skeletal structure such as disulfide bonds between amino acids 19 and 35 as well as 8 and 25 in addition to the planar binding face (mostly aromatic amino acids, i.e., residues 5, 31, and 32). Furthermore, the distance between the aromatic amino acids was highly conserved (approximately 10.4 $\AA$ ) (Linder et al. 1995). Most of the previous reports have focused on these conserved region to enhance the binding ability of CBM (Fukuda et al. 2006). In contrast, the variability of amino acids at positions $18,23,26$, and 27 indicates that these residues are not necessary for the main structure of the CBM. Rather, they may have been tuned to obtain an optimal structure to bind to each biomass cellulose for efficient degradation in many microorganisms. Thus, in this study, we focused on the flexible region to obtain optimal combination of CBM for various types of biomass. A yeast mixture with $\mathrm{CBM}$ that were comprehensively mutated at the flexible residues was constructed to select the optimal combination of CBMs for each biomass cellulose, and the comprehensive mutation was confirmed by the rejection of the null hypothesis for each amino acid (Table 3).

The yeast mixture was cultivated and screened in YNB-newspaper medium to obtain the optimal combination of yeasts displaying EG with mutated CBM. The survived yeasts consisted of RTSH yeast and wildtype yeast in a ratio of $1: 46$, and the blended yeast (the combination of RTSH yeast and wild-type yeast) produced 1.48-fold more ethanol than wild-type yeast alone at $6 \mathrm{~h}$. The amount of ethanol produced by RTSH yeast alone was almost equal to that produced by wildtype yeast alone at $6 \mathrm{~h}$. Interestingly, RTSH yeast produced 1.21-fold more ethanol than wild-type yeast at $24 \mathrm{~h}$ (Figure 4). The higher fermentation rate of RTSH yeast indicates that the saccharification of cellulose by RTSH yeast might be faster than that by wild-type yeast. Thus, CBM with RTSH mutation is probably 


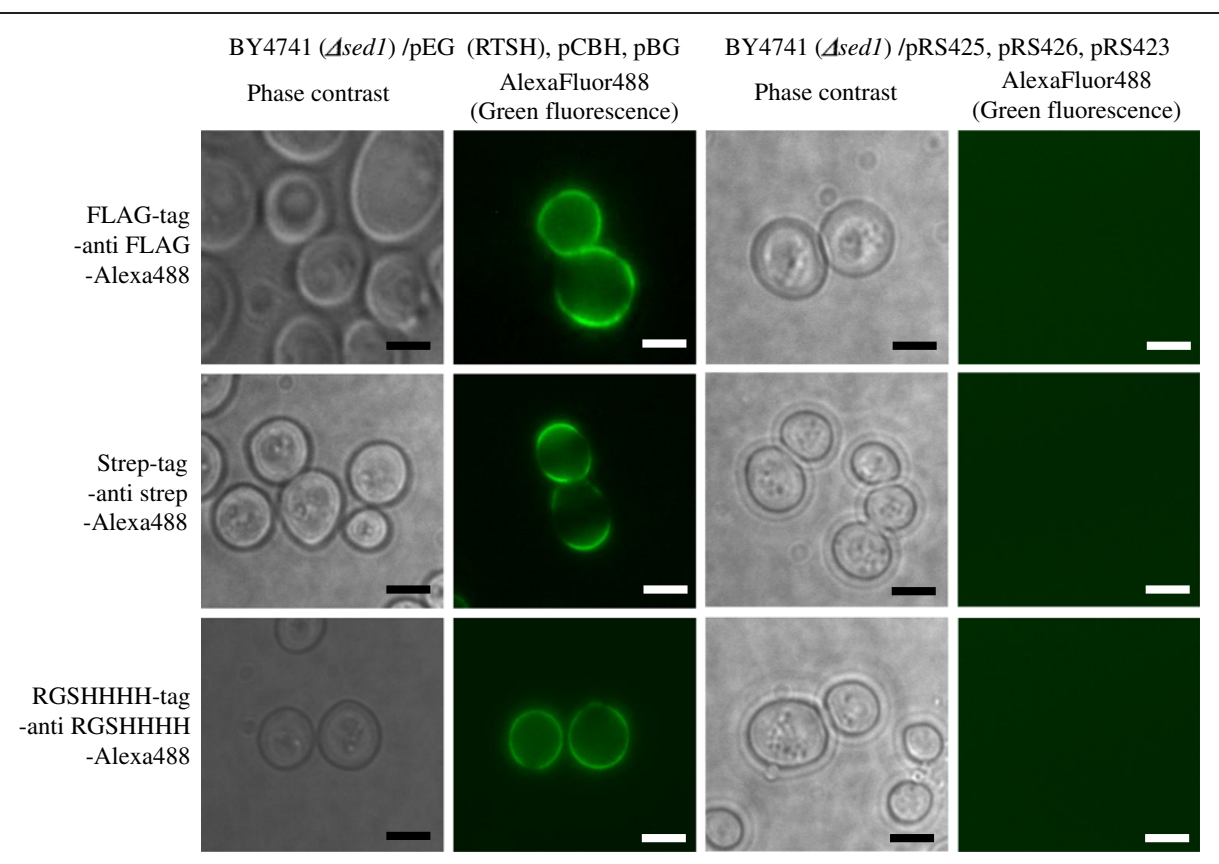

Figure 3 Fluorescence observation of RTSH yeast, harboring EG with CBM having RTSH mutation, CBH, and BG, after immunofluorescence labeling. Yeast cells were labeled with the following antibodies: mouse monoclonal anti-FLAG M2 antibody for EG (upper column), mouse monoclonal StrepMAB-Classic antibody for CBH (middle column), mouse monoclonal anti-RGS-His antibody for BG (lower column), and Alexa Fluor 488 anti-mouse immunoglobulin. The left column represents RTSH yeast and the right column represents yeast harboring pRS423, pRS425, and pRS426 (control vectors). Phase-contrast micrographs are presented in the left column and fluorescence micrographs are presented in the right column. The scale bar is $5 \mu \mathrm{m}$.

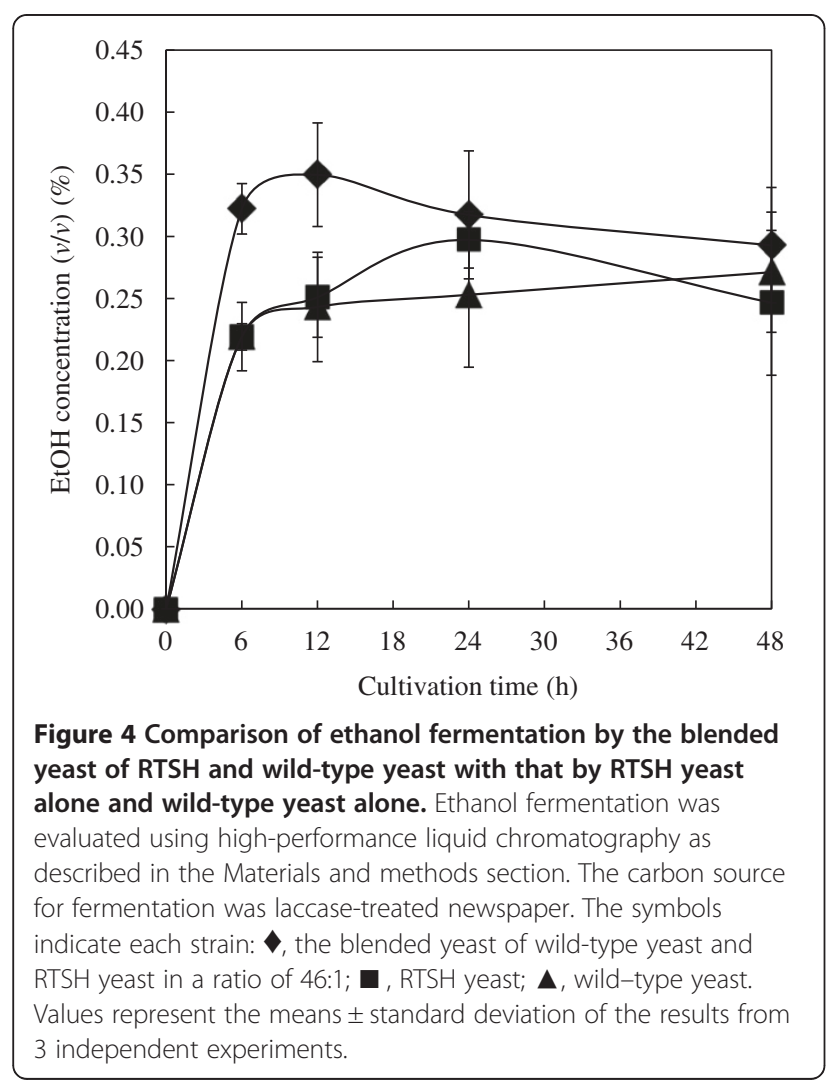

more efficient than wild-type CBM for partial degradation. Attractively, only 1 RTSH yeast was obtained during the selection process, in contrast to 46 wildtype yeasts, which indicates that RTSH yeast could have helped wild-type yeast to degrade the cellulose of newspaper in the early phase of cellulose degradation. Then, the screening was also performed on hydrothermally processed rice straw, and RTSH yeast (N18R, S23T, S26S, and T27H), RPQA yeast (N18R, S23P, S26Q, and T27A), wild-type yeast, and RPLA yeast (N18R, S23P, S26L, and T27A) were obtained in a ratio of 10:7:2:1 (data not shown). The difference in composition depending on the substrate could be due to variation of the cellulose structure in different types of biomass, indicating the feasibility of using this screening system to obtain the optimal combination of CBMs.

The present study is the first to identify flexible residues in family $1 \mathrm{CBMs}$, to construct a selection system for the optimal combination of CBMs of EGs for each type of biomass using a yeast mixture with comprehensively mutated CBM, and to demonstrate improved cellulose saccharification and ethanol fermentation by the selected displaying yeast. Our technique in vitro may be used to easily select the optimal combinations of EGs, like cellulosomes in vivo (Han et al. 2003; Nataf et al. 2010), for various types of biomass. 


\section{Competing interests}

The authors declare that they have no competing interests.

\section{Authors' contributions}

$M U, K K$, and AN participated in the design of this study. MU and KK supervised this study and edited manuscript. AN and JB performed the experimental work, analyzed the data, and drafted the manuscript. All authors read and approved the final manuscript.

\section{Acknowledgement}

This work was supported by a research fellowship from the Japan Society for the Promotion of Science for Young Scientists.

Received: 10 August 2012 Accepted: 21 September 2012

Published: 23 October 2012

\section{References}

Bouaid A, Martínnez M, Aracil J (2010) Biorefinery approach for coconut oil valorization: a statistical study. Bioresource Technology 101:4006-4012

Chandel AK, Singh QV (2011) Weedy lignocellulosic feedstock and microbial metabolic engineering: advancing the generation of 'Biofuel'. Applied Microbiology and Biotechnology 89:1289-1303

Ferreira S, Gil N, Queiroz JA, Duarte AP, Domingues FC (2010) Bioethanol from the Portuguese forest residue Pterospartum tridentatum - An evaluation of pretreatment strategy for enzymatic saccharification and sugars fermentation. Bioresource Technology 101:7797-7803

FitzPatrick M, Champaqne P, Cunninqham MF, Whitney RA (2010) A biorefinery processing perspective: treatment of lignocellulosic materials for the production of value-added products. Bioresource Technology 101:8915-8922

Fujita Y, Ito J, Ueda M, Fukuda H, Kondo A (2004) Synergistic saccharification, and direct fermentation to ethanol, of amorphous cellulose by use of an engineered yeast strain codisplaying three types of cellulolytic enzyme. Applied and Environmental Microbiology 70:1207-1212

Fujita Y, Takahashi S, Ueda M, Tanaka A, Okada H, Morikawa Y, Kawaguchi T, Arai M, Fukuda H, Kondo A (2002) Direct and efficient production of ethanol from cellulosic material with a yeast strain displaying cellulolytic enzymes. Applied and Environmental Microbiology 68:5136-5141

Fukuda T, Ishikawa T, Ogawa M, Shiraga S, Kato M, Suye S, Ueda M (2006) Enhancement of cellulase activity by clones selected from the combinatorial library of the cellulose-binding domain by cell surface engineering. Biotechnology Progress 22:933-938

Gerngross TU, Slater SC (2000) How green are green plastics? Scientific American 283:37-41

Ge X, Burner DM, Xu J, Philips GC, Sivakumar G (2011) Bioethanol production from dedicated energy crops and residues in Arkansas, USA. Biotechnology Journal 6:66-73

Han SO, Yukawa H, Inui M, Doi RH (2003) Regulation of expression of cellulosomal cellulase and hemicellulase genes in Clostridium cellulovorans. Journal of Bacteriology 185:6067-75

Hess M, Sczyrba A, Egan R, Kim TW, Chokhawala H, Schroth G, Luo S, Clark DS, Chen F, Zhang T, Mackie Rl, Pennacchio LA, Tringe SG, Visel A, Woyke T, Wang Z, Rubin EM (2011) Metagenomic discovery of biomass-degrading genes and genomes from cow rumen. Science 331:463-467

Ito H, Fukuda Y, Murata K, Kimura A (1983) Transformation of intact yeast cells treated with alkali cations. Journal of Bacteriology 153:163-168

Kobori H, Sato M, Osumi M (1992) Relationship of actin organization to growth in the two forms of the dimorphic yeast Candida tropicalis. Protoplasma 167:193-204

Kuroda K, Shibasaki S, Ueda M, Tanaka A (2001) Cell surface engineered yeast displaying a histidine oligopeptide (hexa-His) has enhanced adsorption of and tolerance to heavy metal ions. Applied Microbiology and Biotechnology 57:697-701

Kuroda K, Ueda M (2011) Cell surface engineering of yeast for applications in white biotechnology. Biotechnology Letters 33:1-9

Lin Y, Tsumuraya T, Wakabayashi T, Shiraga S, Fujii I, Kondo A, Ueda M (2003) Display of a functional hetero-oligomeric catalytic antibody on the yeast cell surface. Applied Microbiology and Biotechnology 62:226-232

Linder M, Mattinen ML, Kontteli M, Lindeberg G, Ståhlberg J, Drakenberg T, Reinikainen T, Pettersson G, Annila A (1995) Identification of functionally important amino acids in the cellulose-binding domain of Trichoderma reesei cellobiohydrolase I. Protein Science 4:1056-64
López JAS, Li Q, Thompson IP (2010) Biorefinery of waste orange peel. Critical Reviews in Biotechnology 30:63-69

Murai T, Ueda M, Kawaguchi T, Arai M, Tanaka A (1998) Assimilation of cellooligosaccharides by a cell surface-engineered yeast expressing $\beta$ glucosidase and carboxymethylcellulose from Aspergillus aculeatus. Applied and Environmental Microbiology 64:4857-4861

Nakanishi A, Bae JG, Fukai K, Tokumoto N, Kuroda K, Ogawa J, Nakatani M, Shimizu S, Ueda M (2012a) Effect of pretreatment of hydrothermally processed rice straw with laccase-displaying yeast on ethanol fermentation. Applied Microbiology and Biotechnology 94:939-948

Nakanishi A, Kuroda K, Ueda M (2012b) Direct fermentation of newspaper after laccase-treatment using yeast codisplaying endoglucanase, cellobiohydrolase, and $\beta$-glucosidase. Renewable Energy 44:199-205

Nataf Y, Bahari L, Kahel-Raifer H, Borovok I, Lamed R, Bayer EA, Sonenshein AL, Shoham Y (2010) Clostridium thermocellum cellulosomal genes are regulated by extracytoplasmic polysaccharides via alternative sigma factors. Proceedings of the National Academy of Sciences 107:18646-51

Nishitani T, Shimada M, Kuroda K, Ueda M (2010) Molecular design of yeast cell surface for adsorption and recovery of molybdenum, one of rare metals. Applied Microbiology and Biotechnology 86:641-648

Shibasaki S, Ueda M, lizuka T, Hirayama M, Ikeda Y, Kamasawa N, Osumi M, Tanaka A (2001) Quantitative evaluation of the enhanced green fluorescent protein displayed on the cell surface of Saccharomyces cerevisiae by fluorometric and confocal laser scanning microscopic analyses. Applied Microbiology and Biotechnology 55:471-475

Ståhlberg J, Johansson G, Pettersson (1991) A new model for enzymatic hydrolysis of cellulose based on the two-domain structure of cellobiohydrolase I. Nature 9:286-290

Stöcker M (2008) Biofuels and biomass-to-liquid fuels in the biorefinery: catalytic conversion of lignocellulosic biomass using porous materials. Angewandte Chemie (International Ed in English) 47:9200-9211

Todaka N, Lopez CM, Inoue T, Saita K, Maruyama J, Arioka M, Kitamoto K, Kudo T, Moriya S (2010) Heterologous expression and characterization of an endoglucanase from a symbiotic protist of the lower termite, Reticulitermes speratus. Applied Biochemistry and Biotechnology 160:1168-1178

Washida M, Takahashi S, Ueda M, Tanaka A (2001) Spacer-mediated display of active lipase on the yeast cell surface. Applied Microbiology and Biotechnology 56:681-686

Wu L, Arakane M, Ike M, Wada M, Takai T, Gau M, Tokuyasu K (2011) Low temperature alkali pretreatment for improving enzymatic digestibility of sweet sorghum bagasse for ethanol production. Bioresource Technology 102:4793-4799

Ye K, Shibasaki S, Ueda M, Murai T, Kamasawa N, Osumi M, Shimizu K, Tanaka A (2000) Construction of an engineered yeast with glucose-inducible emission of green fluorescence from the cell surface. Applied Microbiology and Biotechnology 54:90-96

doi:10.1186/2191-0855-2-56

Cite this article as: Nakanishi et al:: Construction of a novel selection system for endoglucanases exhibiting carbohydrate-binding modules optimized for biomass using yeast cell-surface engineering. AMB Express 2012 2:56.

\section{Submit your manuscript to a SpringerOpen ${ }^{\odot}$ journal and benefit from:}

- Convenient online submission

- Rigorous peer review

- Immediate publication on acceptance

- Open access: articles freely available online

- High visibility within the field

- Retaining the copyright to your article

Submit your next manuscript at $>$ springeropen.com 